\title{
Multisensory Games for Dyslexic Children
}

\author{
James Ohene-Djan and Rahima Begum \\ Goldsmiths College, University of London \\ New Cross, London SE14 6NW \\ j.djan@gold.ac.uk
}

\begin{abstract}
A significant problem faced by dyslexic children is a lack of learning technologies designed to help children learn in settings when there is no personal teaching assistance. This paper presents an online learning technology that utilises Multisensory teaching, and interactive gaming techniques to provide dyslexic children with an engaging learning environment within which to identify where their mistakes in reading, writing and arithmetic lie. It is hoped that by providing this example of a interactive learning system specifically created for the needs of dyslexic children will raise awareness and understanding among learning technology software developers and practitioners.
\end{abstract}

\section{Introduction}

Dyslexia is a neurological problem that covers a wide range of reading disabilities [1]. Although still not fully understood it is recognised that what most dyslexics have in common is a difficulty in grasping the shapes of letters and then relating those shapes to the sounds that the letters symbolize. Dyslexics often reverse the order of the letters in a word or even leave them out completely. Other effects of Dyslexia include difficulties in memory, organisation, numeracy [2], time management, low self-esteem and a lack of selfconfidence [3].

In the UK it is estimated that one in ten children exhibit some form of dyslexia and it is estimated that $80 \%$ of people diagnosed with learning difficulties are also dyslexic [4]. A proven approach to helping children overcome their dyslexia is to enable them to practice weaknesses and to identify where their mistakes in reading, writing and arithmetic lie. Using such an approach it has been shown that dyslexic children can learn subject matter just as well as nondyslexic children. However, to achieve this often the child must be taught using alternative teaching strategies and be supported by a personal assistant to help identify mistakes and monitor progress.

A significant problem faced by dyslexic children is a lack of learning technologies designed to help identify weaknesses in the absence of personal assistance in settings such as in home. The vast majority of the software available is designed to be used in school settings with teachers controlling the pace and form of learning. Furthermore, as many schools find it difficult to fund support teachers to provide personal assistance this software is sometimes not used or available in school settings.

Support for dyslexic children to study at home generally takes the form of practice worksheets containing a range of study exercises realized as multiple choice questions, training exercises and to open questions. However, such worksheets are often criticised for there lack of interactivity and failure to engage the child so as to motivate them to learn.

This paper presents The Dyslexia Activity System (DAS), an online learning technology that utilises Multisensory teaching [5] to provide dyslexic children with an engaging learning environment within which to identify where their mistakes in reading, writing and arithmetic lie. Although designed for young dyslexic children in key stage one of the UK's education system, DAS can also be used for non-dyslexic children to improve on sum numeracy, word building, memory and sequencing skills.

\section{Background}

Multisensory teaching can be understood to be a method of teaching in which strengths that dyslexic children appear to have over non-dyslexic children, such as extra creativity and stronger sensory receptors (tactile/touch), are implemented as part of the teaching process. Originally proposed by a collective of learning difficulty specialists, teachers and researchers 
in the late 1920s [6], and significantly developed in the 1970's by Gillingham \& Stillman [7], its aim is to find out what triggers productive learning in children with learning difficulties and how to develop teaching strategies that supported their learning. Recent Studies from the National Institutes of Child Health and Human Development have shown that multisensory teaching is the most effective teaching method for children with learning difficulties and is a crucial development tool for children with dyslexia [8].

Multisensory teaching consists of a string of Multisensory strategies that include techniques for linking eyes, ears, voice, and hand movements to symbolic learning. The approach taken is to try and engage as many sensory receptors in the learning process as possible, since it is argued that on many occasions, children with learning difficulties appear to have extra receptive sensors that can be used to bookmark learning events within their the memory.

An example of a Multisensory teaching approach is to use graphics and strong colours to make associations between shape, letters, words and numbers that relate to the same topic. Multisensory teaching also uses tactile objects and touching for emphasize during study. For example often dyslexic children find it difficult to distinguish the letters "b" from " $d$ ". So the child is asked to draw a giant letter "b" on the carpet with his finger feeling the texture, since the child's sensory receptors are perceived as strong it is hoped their memory system will recall the "feeling and shape of the letter" and thus make identifying it easier in the future.

Multisensory teaching approaches can also be applied in two dimensions though the use of pictorial representations that enable children to form associations with letters, words and numbers. For example, the letter " $A$ " can be represented as a string of apples connected together to make the letter. Multisensory teaching is very open and flexible approach that aims to work in different ways for different individuals, which is precisely what children with dyslexia require.

Often children with dyslexia also have difficulties with in various areas of mathematics, for example multiplication, fractions, decimals, percentages, ratio and statistics [2]. Using Multisensory teaching approaches to the design of mathematical exercises has proven to successful in improving individual abilities by reinforcing the relationship of numbers to actual quantities. [9].

Broadly, educational learning games introduce strategies and elements of games into a learning system's structure and modes of interaction.
Educational learning games usually comprise of all the elements that traditional games require such as rules, challenges interactivity and creativity. When designing educational learning games for children it is important to use graphics, symbols, animation and relatively low amounts of text on the user interface. Such considerations help to engage the child to interact with the games, make them more fun and interesting which all contributes to the environment of learning. [10]

Educational learning games have proved an effective way of motivating children with learning disabilities to learn. Children tend not to see learning as a chore and are often more willing to have an attempt at a game then a worksheet. In the case of Dyslexic children activities involving gaming strategies can be used as a reinforcement factor that allows children to see mistakes in reading, writing and arithmetic as challenges to completing the game. ICT and games can be used to present an active learning environment, which is crucial for children with learning difficulties such as dyslexia. [11].

\section{Dyslexia Activity System}

The Dyslexia Activity System (DAS) reported in this paper is an online Multisensory teaching system requiring high levels of child interactivity and engagement. The system is designed to support learning though a series of gaming techniques and association reinforcement exercises. These activities have been designed to help capture a child's attention and motivate them to learn through a fun environment. An aim of the system is to motivate children to build and strengthen their keys skills such as numeracy and literacy though interactive play. Although the primarily designed for dyslexic children, it is hoped that the use of Multisensory teaching approach embodied within DAS will also benefit non- dyslexic children and those with other learning needs.

\subsection{Overview of design of DAS}

DAS presents an alternative approach to traditional paper worksheets by presenting a set of Multisensory teaching exercises as interactive games tailored to the child's needs. Each game has been designed using minimal user textual input and provides the child with control over the learning process. By giving the child control of the application it is hope to develop selfconfidence and drive to succeed.

Paper worksheets make it difficult for the child to see their progress, which can in effect motivational 
levels. IN DAS an animated interactive score bar details a child progress based on numbers mistakes in reading, writing and arithmetic. The system has been designed in such a way as to allow the dyslexic child to attempt an exercise or choice within an exercise multiple times so that they may correct mistakes. Correct scores are reinforced with motivational prompts and these are used show the child the benefits of their hard work. Although DAS has been specifically designed for young dyslexic children in key stage one of the UK's education system, it can also be used for young children regardless of whether children have dyslexia because children can improve on sum numeracy, word building, memory and sequencing skills.

A criticism of some ICT systems used by dyslexic children is that their interfaces are too complicated and have too much text that confuses users and generates cognitive overload. DAS has been created using a simple clear, bold interface, supporting the customisation of colour schemes to minimise the effects of dyslexic. These colour schemes play a crucial role for creating a system for dyslexic individuals, since strong colours and complicated patterns can be distracting. DAS uses light pastel colours, which helps lift the text of the page and becomes easier to read for dyslexic children.

\subsection{Multisensory teaching in DAS}

Within DAS Multisensory teaching of exercises has been implemented as a set of activities. Activity one is a letter recognition exercise, which helps develop reading, organizational and memory skills. Activity two helps children develop number recognition skills. Activity three is a number sequencing exercise designed to help develop memory skills and numeracy. Activity four is a vowels and phonics exercise, which helps the child in developing language skills and Activity five is a sequencing and character recognition exercise is designed to coach colour and shape recognition.

Grouping Strategy is a words recognition and organization activity. The purpose of the activity is to help the user understand what the words means and what category it belongs in. By sorting through the words it also helps the child build on their organizational skills.

Fun Sums is a numeric and mathematic based activity aimed at helping children to understand how to solve sums and simple subtractions and multiplications.

\subsection{Examples of multisensory exercises}

Figure 1 shows an example Activity one level letter recognition exercise. The exercise uses symbolic clues, colours and the mapping of associations to reinforce the learning experience. This exercise is used to help a child develop a range of reading skills.

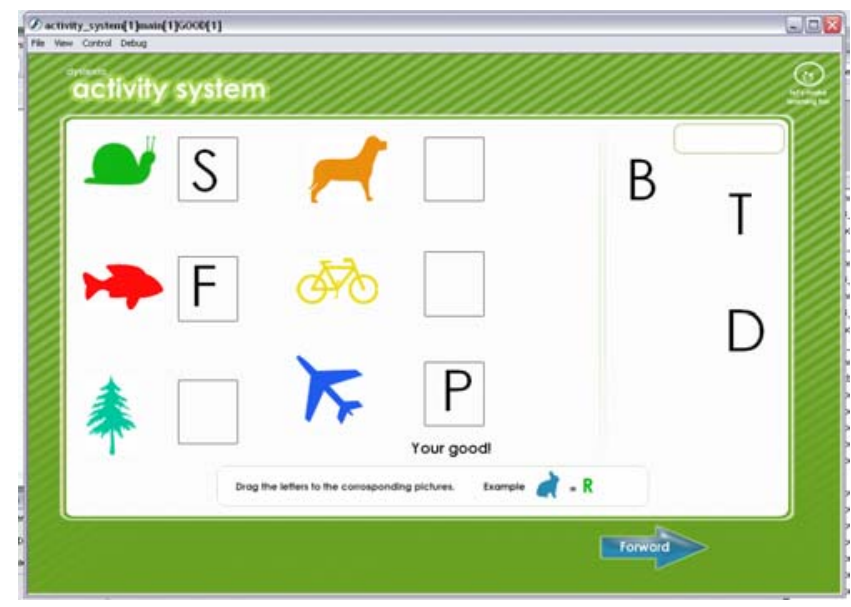

Figure 1. Letter recognition exercise

Figure 2 shows an example number recognition exercise. The exercise is designed to reinforce the shapes associated with numbers and number sequences.

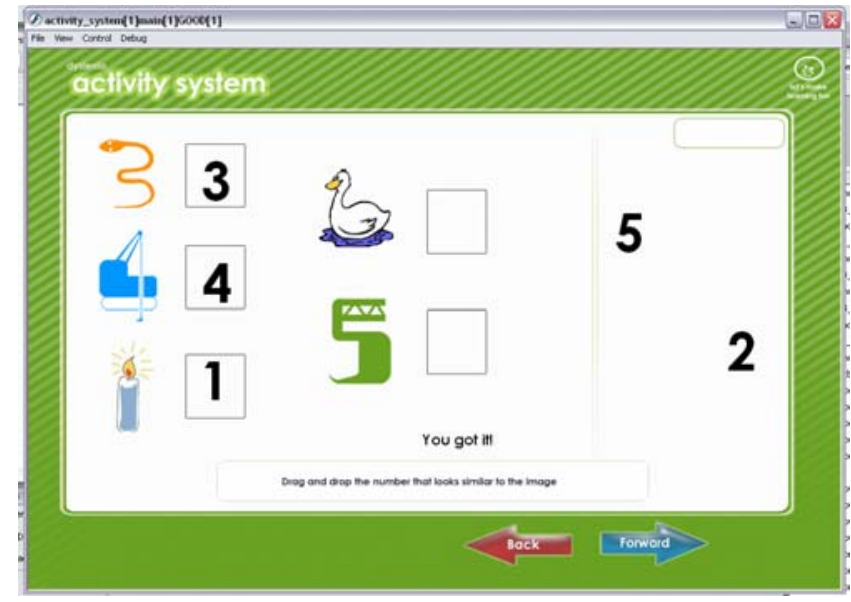

Figure 2. Number recognition exercise

Figure 3 shows an activity exercise called the "Vowels in a Basket". This exercise is vowels and phonics exercise designed to help children in developing language and organizational skills. This is accomplished via the use of multisensory teaching and the use of pictures as clues to help the child. The 
activity uses strong colourful images to make the working environment lively and fun.

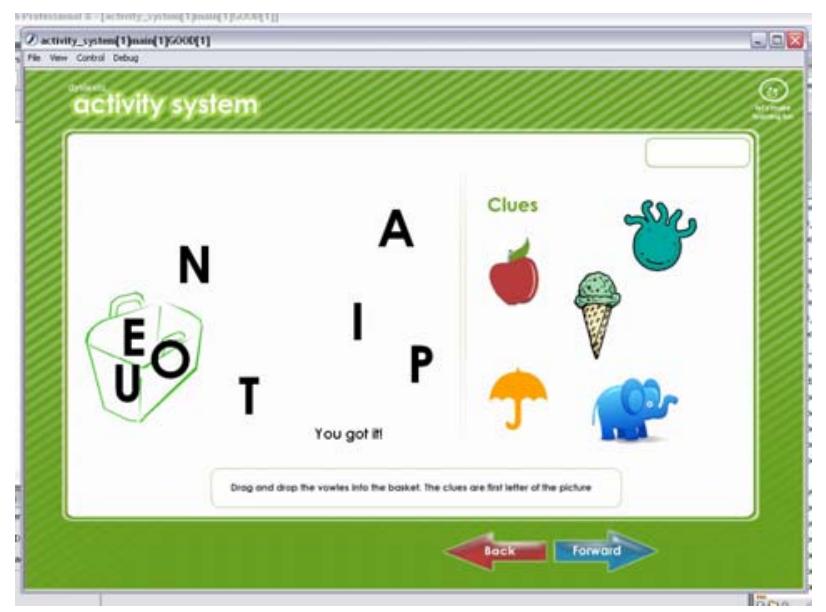

Figure 3. Vowels in a basket

Figure 4 shows an exercise designed to support multisensory teaching of sequences and shape recognition. The exercise uses a clear layout with simple instructions together with colour and shape recognition supported by a “drag and drop” interface.

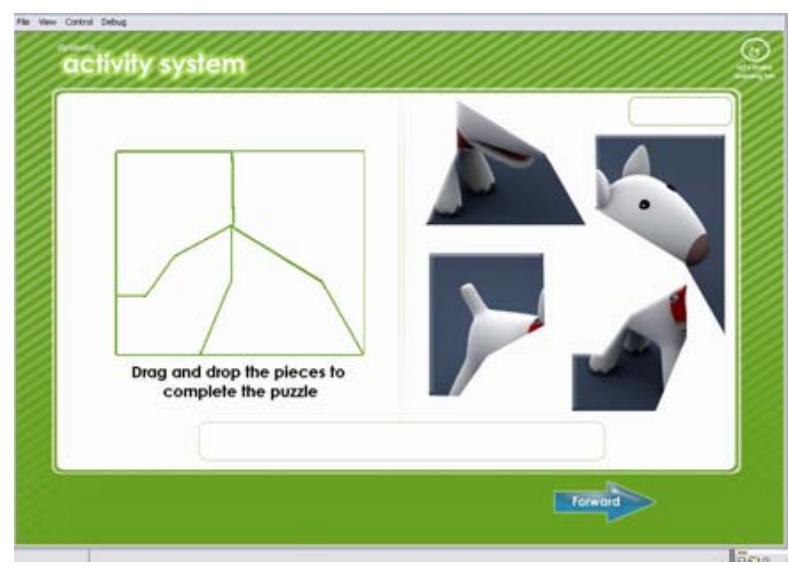

Figure 4. Shape and recognition exercise

Figure 5 depicts a grouping strategy exercise designed to teach word recognition skills. The purpose of the activity is to help the child understand what the words mean and what category they belongs to. By sorting through the words it is hope that children will also improve their organizational skills.

To make the activity easy to understand, each exercise of this type is supported by a brief description. To make it easier for children to distinguish the family groupings of words each group is designated its own colour. The child is required to drag and drop all the corresponding words into the correct family. Attempts, mistakes and results are shown in a score bar.

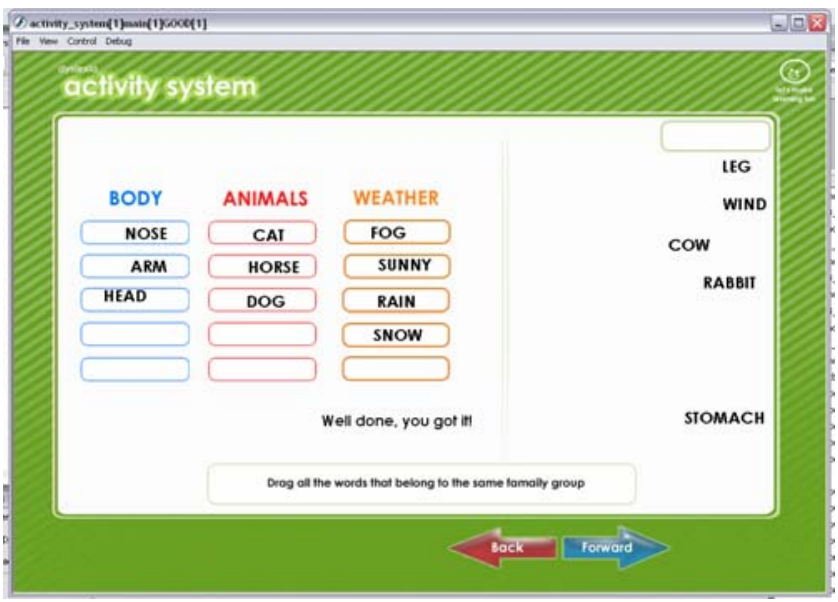

Figure 5. Group strategy exercise

Figure 6 shows "Fun Sums", a numeric and mathematic based activity designed to teach the fundamentals of adding, subtraction and multiplication. The current question is highlighted while the rest of the questions are faded. To assist the child in resolving the questions, animations are used. For example the question uses an animation of oranges falling into a basket to ask what is $4+4$. Four oranges are in the basket and the animation shows the other four falling into it. This helps in breaking the question down for the child and makes them aware of how to solve the question. In essence the animation is a support teacher.

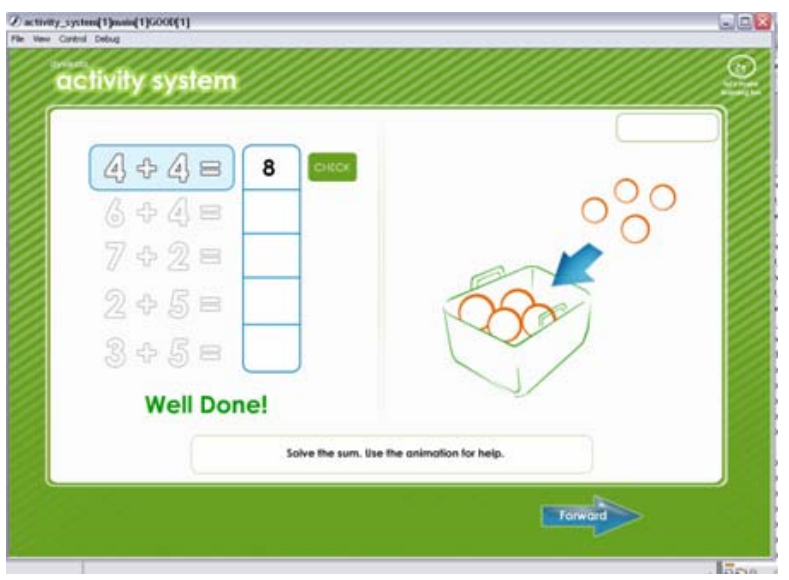

Figure 6. Fun Sum exercise 


\section{Related Work}

Worksheets are the most common form educational learning materials used to teach dyslexic children. Worksheets are used to "drill and test" children and provide them with skill building practice. Since they are fairly low cost to produce, worksheets play a major role in the schools and are used by teachers to guide learning. Worksheets usually contain learning material on letters, numbers, and shape identification and are very easy to obtain from the Internet or local educational authorities.

A significant drawback of worksheets is that they are often viewed as a chore or even punishment that does not engage or stimulate the children. Studies in children's behaviour and learning have shown constantly that children learn better through active involvement with real tactile objects and with a combination of multisensory teaching techniques. A further drawback is that children with learning difficulties, especially dyslexic children often struggle digesting material on worksheets due to being compacted with text and with complicated formats [12].

Wordsworks is a commercially available multisensory teaching tool designed for adults (www.wordswork.co.uk) that embodies design principles similar to those reported in this paper. Wordswork is an interactive support system that contains a range of activities and tools. Some of the topics that Wordswork addresses include: improving reading, spelling, punctuation, essay writing, mind mapping, punctuation, handwriting and oral presentations. The system is designed to encourage the adults to practise strategies and suggest approaches to make their learning more manageable [13].

Even though the existence of Wordsworks demonstrates the potential of using ICT to teach using a multiseronry approach it does not lend itself well to the teaching needs of children. In particular, it has been suggested that its user interface and content are inappropriate for teaching under $10 \mathrm{~s}$ and that the levels and type of interactively is not appropriate for children. Through the development of DAS it is hoped that it can contribute towards the development of a commercial multiseronry teaching tool designed specifically for children.

\section{Conclusions}

We have presented an online learning technology called DAS that utilises Multisensory teaching, and interactive gaming techniques to provide dyslexic children with an engaging learning environment. We hope by demonstrating interactive learning system specifically designed for the needs of dyslexic children we will raise awareness and understanding among learning technology practitioners.

\section{References}

[1] L. Peer and G. Reid Introduction to dyslexia: What is dyslexia, chapter 2 pages 13-14. Part of the British Dyslexia association. ISBN 1853469645 (2003).

[2] J. Herold. Dyslexia and math's. On-line article. Web link: www.dyslexia-parent.com/mag43.html (Feb 2003).

[3] M.J. Snowling, Dyslexia is Not a Myth. On-line article web link: www.bdadyslexia.org.uk/news17.html University of York. (2005).

[4] B. Hornsby, Overcoming Dyslexia: A Straightforward Guide for Families and Teachers ISBN-10: 0091813204 Vermilion; 3Rev Ed edition (1996).

[5] C. Singleton, Understanding dyslexia. Web link: www.portobello.edin.sch.uk/LSSN/LSSN2.html. University of Hull. (2000).

[6] H. Schupack and B. Wilson, The " $R$ " Book, Reading, Writing \& Spelling: The Multisensory Structured Language Approach, Baltimore. The International Dyslexia Association’s Orton Emeritus Series. (1997).

[7] J.R.. Birsh. Multisensory Teaching of Basic Language Skills, Second Edition, chapter 1, page 27. ISBN 1-55766676-8. (2005).

[8] J. Bradford What causes dyslexia. Web link: www.dyslexia-parent.com/mag24.html, (2003).

[9] L. Trie, Stanford Report Remediation training improves reading ability of dyslexic children. On-line article. (2003).

[10] W. Goldup. Dyslexia Review: The journal of the dyslexia institute Guild, volume 15, Number 2 pages 14, 19. ISSN 0308 6275. (2004).

[11] C. Singleton and J. Hutchins. Computer and literacy skills: Reading Software, chapter 5 pages 45. ISBN 1872653 02 2. (1991).

[12] P. Patten, School of Human Resources and Family Studies, University of Illinois Worksheets: Good or Bad?. Web link: www.nncc.org/Curriculum/dc26_worksheet.html, (1996).

[13] E. Morgan Wordswork, Web link: www.dyslexia-adults.com/wordswork.htm, (2000). 